\title{
Treatment of acute uncomplicated cystitis. a clinical review
}

\begin{abstract}
Urinary tract infection (UTI) is the most common bacterial infection encountered in the ambulatory care setting. Many women have had at least one symptomatic UTI, and some of them have had mutiple recurrences. ${ }^{1-3}$ In 2007, a nationwide Spanish poll was conducted on 6,545 randomized women and revealed that $37 \%$ had at least one episode of lower UTI, and $32 \%$ of them had suffered two or more episodes. ${ }^{4}$ So, since UTI in females is an infection so frequent in the communiy, each improvement of management will have a high impact not only on the quality of live of the individual but also on the health system as a whole. Acute cystitis is an infection of the bladder and is referred to as a lower UTI. Pyelonephritis is infection of the kidney, the upper urinary tract; is more severe and can occur in conjunction with acute cystitis. In practice, it is sometimes difficult to make a clear distinction between these two clinical síndromes. ${ }^{3-5}$
\end{abstract}

Keywords: UTI, urinary tract infection; ESCMID, european society for microbiology and infectious diseases; IDSA, infectious diseases society of america; ESBL, extendedspectrum beta lactamase
Volume I Issue 2 - 2017

\author{
Luis Aliaga, Manuela Moreno, Ismael Aomar, \\ Susana Moya, Ángel Ceballos, Pilar Giner \\ Departamento de Medicina, Universidad de Granada, Spain
}

Correspondence: Luis Aliaga, Departamento de Medicina, Universidad de Granada, Spain, Tel 61828393I, Email laliaga@ugr.es

Received: August 23, 2017 | Published:September 22, 2017

\section{Introduction}

The incidence of cystitis is much higher than that of pyelonephritis. Uncomplicated UTIs are episodios of acute cystitis or pyelonephritis when it occurs in a healthy premenopausal, non-pregnant woman with no known urological abnormalities (structural or functional). ${ }^{1-5}$ UTI that do not meet these criteria is considered complicated. This review is focused on management of acute uncomplciated cystitis. Although some experts may consider "uncomplicated" those UTIs occurring in postmenopausal women or in well-controlled diabetic women without urological sequelae ${ }^{6}$ a discussion of specific management of these groups is beyond the scope of this article. In 2011, the Infectious Diseases Society of America (IDSA) in collaboration with the European Society for Microbiology and Infectious Diseases (ESCMID) published a clinical practice guideline on the treatment of women with acute uncomplicated cystitis and pyelonephritis.

This document has become an invaluable reference in the management of UTI. In this guideline the resistance pattern among the uropathogens along with the potential for collateral damage of the antimicrobials were considered as important factors in making optimal treament choices; and, thus were reflected in the rankings of recommendations. With respect to antimicrobial susceptibility of uropathogenic bacteria, this guideline pointed out two important facts:

a. Antibiotic resistance of uropathogens is increasing over the past years.

b. The resistance pattern shows a considerable geographic variability between countries and even regions.

For example, in an international survey of the antimicrobial susceptibility of uropathogens from uncomplicated UTI, the overalll resistance rates among uropathogens were usually higher in Portugal and Spain than in other European countries. ${ }^{7}$ Thus, as it was recognized in theseguidelines, "a specific treatment recommendation may not be universally suitable for all regions or countries". ${ }^{1}$ On the other hand, the IDSA advised the need for resistance surveillance studies over time at local practice and/or health care system levels for sustained optimization of empirical therapy. Therefore, the guidelines have to be periodically revised because of the continuously evolving resistance of uropathogens to antimicorbial agents, developtment of new agents, and production of studies showing superiority/inferiority of agents, as well as studies redefining appropriate lengths of therapy. In this regard, the Spanish Society of Clinical Microbiology and Infectious Diseases (SEIMC) has recently updated a clinical guideline on the diagnosis and treatment of UTI. ${ }^{8}$ Our review is intended to incorporate the recent knowledge in these aspects. To do so, a consideration of antimicrobial resistance and collateral damage must be done.

\section{Antimicrobial resistance}

The microbial spectrum of uncomplicated cystitis and pyelonephritis consists mainly of Escherichia coli (75-95\%), with occasional other species of Enterobacteriaceae, such as Proteus mirabilis and Klebsiella pneumoniae, and Staphylococcus saprophyticus (particularly in young females who are sexually active). ${ }^{1-11}$ Other gram-negative and gram-positive species are rarely isolated in uncomplicated UTI. Moreover, a recent study has shown that in women with acute uncomplicated cystitis, enterococci and group $B$ streptococci isolated from midstream urine were often not found in bladder urine obtained simultaneously by catheterization. ${ }^{12}$ Therefore, antimicrobial susceptibilty patterns of $E$. coli in particular should be taken into account in empirical antimicrobial selection for uncomplicated UTI. Resistance pattern of E. coli strains responsible for uncomplicated UTI changes considerably between regions and countries. Thus, a specific recommendation may not be applicable for all regions or countries. Active surveillance studies of in vitro susceptibility of uropathogens in women with uncomplicated cystitis are advisable in making decisions about empirical therapy.

Local resistance rates reported in hospital antibiograms may be biased by cultures of samples from inpatients or those with complicated infection and may not predict susceptibilities in women with uncomplicated community-acquired infection, in whom resistance rates tend to be lower. ${ }^{14}$ Thus, prospective and unbiased resistance surveillance studies of uropathogens, preferably at the 
local practice, is essential to guide empirical antimicrobial decisions in uncomplicated UTI. Studies reporting in vitro susceptibility of E. coli causing uncomplicated UTI in North America and Europe demonstrated considerable geographic variability in susceptibility and a general trend toward increasing resistance. ${ }^{1-15}$ These studies also revealed that, among the antimicrobials in common use for treatment of uncomplicated UTI, nitrofurantoin, fosfomycin and pivmecillinam (the latter not licensed in Sapin) have preserved their in vitro activity in all the countries investigated. ${ }^{1-15}$ Thus, these 3 antimicrobials were considered in the IDSA guidelines appropriate antimicrobials for empirical therapy in most regions. ${ }^{1}$

In Spain, resistance patterns of E. coli also varies between regions. In a survey of 515 women with uncomplicated cystitis, ${ }^{4-10}$ susceptibility rates of $E$. coli were the following: nalidixic acid, $73 \%$; ciprofloxacin, $89 \%$, fosfomycin, 97\%; nitrofurantoin, 94\%; ampicillin, 35\%; amoxicillin-clavulanic acid, 78\% (18.6\% inermediate resistance and 3.6\% resistant), cefuroxime axetil, $75 \%$ $(22.9 \%$ intermediate resistance and $1,7 \%$ resistant); mecillinam, $94 \%$, and cotrimoxazole, $66 \%$. These results were corroborated in a study by Cuevas et al. ${ }^{16}$ These data indicate the very high level of resistance among the uropathogenic E. coli in Spain, which invalidate many antimicrobials for empirical use. Epidemiological studies have shown that either the use of trimethoprim-sulfamethoxazole or travel outside United Sates in the preceding 3-6 months are independent risk factos for resistance to this drug in women with uncomplicated cystitis. ${ }^{1}$ In fact, a recent paper from Greek has shown that the use of co-trimoxazol or a fluroquinolone in the previous three months was significantly associated with the isolation of a strain of E. coli resistant to co-trimoxazol or ciprofloxacin, respectively. ${ }^{17}$

Treatment of acute uncomplicated cystitis is usually empirical. As the population resistance prevalence of a specific agent increases, one can asume that the likelihood of failure may outweigh the benefit of using a particular drug empirically. In the case of trimethoprimsulfamethoxazole, a resistance prevalence of $20 \%$ is the threshold at which the agent is no longer recommended for treatment of acute cystitis. The threshold of $20 \%$ is based on expert opinion derived from clinical, in vitro, and mathematical modeling studies. ${ }^{1-18}$ There are insufficient data for other cystitis antimicrobials to recommend resistance thresholds, and the decision to avoid a particular antibiotic will vary in an individual practitioner basis. ${ }^{1}$

\section{Collateral damage}

"Collateral damage" is a term to describe ecological adverse

Table I: Level of scientific evidence and grades of recommendation. effects of antimicrobial therapy, such as the selection of drug-resistant organisms and colonization or infection with multidrug-resistant organisms. Ecological adverse effects have been observed with use of broad spectrum cephalosporins and fluoroquinolones. Use of broad spectrum cephalosporins has been linked to subsequent infeciton with vancomycin-resistant enterococci, extended-spectrum beta-lactamase (ESBL)-producing K. pneumoniae, beta-lactam-resistant Acinetobacter species, and Clostridium difficile. ${ }^{1}$ Use of fluoroquinolones has been linked to infection with methicillin-resistant Staphylococcus aureus, and with increasing fluoroquinolone resistance in Gram-negative bacilli, such as Pseudomonas aeruginosa. ${ }^{1}$ It has been suggested that the preserved in vitro susceptibility of $E$. coli to nitrofurantoin and fosfomycin over many years of use migth be due to the only minor collateral damage on normal fecal flora these antimicrobials cause1. In contrast, increased rates of antimicrobial resistance have been observed with antimicrobials that affect the normal fecal flora more significantly, such as trimethoprim, trimethoprim-sulfamethoxazole, quinolones and ampicillin. ${ }^{1}$

Collateral damage merits attention in treatment of actue uncomplicated cystitis. Firstly, because untreated cystitis rarely progresses to symptomatic upper-tract infection or sepsis. ${ }^{1,2}$ On the contrary, the infection may cure in $25-42 \%$ of women without treatment or inappropriate treatment 1 . However, data do not justify witholding antimicrobial therapy in acute cystitis, because treatment with placebo is associated with prolongation of symptoms as well as a small risk of progression to pyelonephritis. ${ }^{1-19}$ Thus, uncomplicated UTI is one of the most common indications for antimicrobial use in medicine. Second, very small collateral damage repeated many times may accumulatively magnify the impact of collateral damage when it occurs. ${ }^{1}$

\section{Methods}

We searched the Spanish and English-language medical literature from Januray 1, 2010 to June 30, 2017, using PubMed database. We used the key word cystitis with the following filters: acute uncomplicated UTI, women, clinical trials, and specific antimicrobials or classes of antimicrobials. We also reviewed bibliographies of the retrieved articles as well as systematic reviews for additional relevant studies. We excluded antibiotics that are currently not available in Sapin, as well as studies that included enrolled patients with complicated UTI. We used the grading scale of the Spanish Society of Clinical Microbiology and Infectious Diseases (SEIMC) for ranking the grades of recommendation (A-E) and for the level of scientific evidence (I-III) ${ }^{8}$ (Table 1).

\section{Level/Grades Definition \\ Level of scientific evidence}

I Evidence obtained from $\geq$ I randomized clinical trial

II

Evidence obtained from $\geq$ I well-designed non-randomized clinical trial, or cohort studies, or case-control-studies, especially if they have been performed in more than one centre

III Evidence obtained from documents or opinions of experts, base don clinical experience or case series

\section{Grades of recommendation}

$\begin{array}{ll}\text { A } & \text { Good evidence to recommend the use of a measure or practice } \\ \text { B } & \text { Moderate evidence to recommend the use of a measure or practice } \\ \text { C } & \text { Poor evidence too recommend the use of a measure or practice } \\ \text { D } & \text { Moderate evidence to discourage the use of a measure or practice } \\ \text { E } & \text { Good evidence to discourage the use of a measure or practice }\end{array}$




\section{Management of acute uncomplicated cystitis}

In women with classic symptoms of cystitis, neither urine culture nor dipstick are necessary for management. ${ }^{2-6}$ Nevertheless, if done, a negative dipstick or microscopic examination for pyuria would raise great suspicion that the diagnosis of cystitis is incorrect. ${ }^{2}$ So, rapid urine test for detection of nitrites and leucocytes have a high negative predictive value in the diagnosis of acute cystitis; thus it could reduce the amount of prescribed antibiotics. For this reason, some guideliens on acute uncomplicated cystitis recommend their systematic use, such as the French, the Scottish and that of the Europena Association of Urology. ${ }^{14}$ In cases of symptoms which do not respond or if they recur after therapy is discontinued, the possibility of upper urinary tract infection should be contemplated and urine culture is indicated. ${ }^{2-6}$ Forcing fluids has been advocated for the therapy of UTI2. Because there is no evidence that forcing fluids improves the results of appropriate antimicrobial therapy, and because continuous hydration is inconvenient, some experts are not in favor of this approach. ${ }^{2}$ Accordingly, forcing fluids in the treatment of acute uncomplicated cystitis should not be recommended (C-III).

The optimal agent for therapy of a patient with acute uncomplicated cystitis depends on a number of factors, such as side effects, cost, and inconvenience of different therapeutic regimens. Each agent may have advantages and disavantages related to its use and the choice of therapy should be made on an individual basis. Appropriate use of antibiotics for urinary tract requires selection and administration of the right dosage of the most suitable antibiotic for a determined time period to eliminate pathogens quickly and successfully. In the past, 7 to 10 days of therapy was routinely recommended for women with cystitis. ${ }^{2-20}$ However, it has become apparent that most women with cystitis can be cured with much shorter courses of therapy and, in fact, many with only a single dose of an antimicrobial agent. The advantages of short-course therapy include a lower cost, better compliance, fewer side effects, and perhaps less intensive selective pressure for the emergence of resistant organisms in gut, urethral, or vaginal flora. ${ }^{2-7}$

Single-dose therapy with certain agents achieves high urinary concentrations that are prolonged for at least 12 to 24 hours and eliminates infection when presumably confined to the bladder. ${ }^{2}$ Historically, regimens such as single oral doses of $3 \mathrm{~g}$ of amoxicillin, one to two double-strength tablets of trimethoprim-sulfamethoxazole, sulfonamides, tetracycline, trimethoprim, nitrofurantoin, fosfomycin, cephalosporins, fluoroquinolones, and a single injection of aminoglucoside were evaluated with high cure rates. ${ }^{2}$ Actually, only fosfmomycin trometamol is approved by the U.S. Food and Drug administration for single-dose ( $3 \mathrm{~g}$ oral powder) teatment of uncomplicated lower UTI. In the same way, a meta-analysis study has shown that antibiotic therapy for 3 days is similar to prolonged therapy (5-7 days) in achieving symptomatic cure for cystitis, while the prolonged treatment is more effective in obtaining bacteriological cure. $^{21}$ The shorter regimen is also associated with fewer side effects and lower costs..$^{21,22}$ Table 2 lists the currently dosage and recommended duration of therapy of some antimicrobials agents in common use for acute uncomplicated cystitis.

\section{Fosfomycin trometamol}

Fosfomycin was discovered in 1969 as a member of a novel class of phosphonic antibiotics. ${ }^{23-26}$ In Spain, fosfomycin is available as disodium fosfomycin for intravenous use and as oral formulations, either as calcium salt and tromethamine salt (synonym trometamol). The latter can be used as single dose and this is the oral form usually prescribed. However, in a recent open-label study from Japan, ${ }^{27}$ it was estimated that fosfomycin calcium, $1 \mathrm{~g}$ administered three times daily for 2 days, provides comparable efficacy to a single-dose treatment of $3 \mathrm{~g}$ fosfomycin tromethamine. Fosfomycin has broad-spectrum bactericidal activity against staphylococci, enterococci, Haemphilus spp, and most enteric Gram-negative bacteria, including 95,5\% of extended-spectrum $\beta$-lacamase (ESBL)-producing $E$. coli. . $^{23-30}$ In addition, $97 \%$ of AmpC-producing $E$. coli isolates obtained by clinical laboratories across Canada from 2007 to 2013 were susceptible to fosfomycin. ${ }^{15}$ Also, strains of $E$. coli producing metallo $\beta$-lactamase are susceptible to fosfomycin. ${ }^{26-28}$

Table 2 Empirical treatment of acute uncomplicated cystitis. Dosages and duration of treatment

\begin{tabular}{|c|c|c|}
\hline Antibiotic & Dosage & Duration(Days) \\
\hline \multicolumn{3}{|l|}{ First-line agents } \\
\hline Fosfomycin Trometamol ${ }^{\mathrm{a}}$ & $3 \mathrm{~g}$ single dose & Single dose \\
\hline Fosfomycin Calcium a,b & I g tid & 2 \\
\hline Nitrofurantoin Macrocrystals ${ }^{\mathrm{a}, \mathrm{c}}$ & $50-100 \mathrm{mg}$ qid & 7-May \\
\hline Pivmecillinam & $400 \mathrm{mg}$ bid & 3 \\
\hline \multicolumn{3}{|l|}{ Second-line agents } \\
\hline Ciprofloxacin & 250- $500 \mathrm{mg}$ bid & 3 \\
\hline Levofloxacin $^{d}$ & $250-500 \mathrm{mg}$ once & 3 \\
\hline Norfloxacin $^{d}$ & $400 \mathrm{mg}$ bid & 3 \\
\hline Ofloxacin ${ }^{d}$ & $200 \mathrm{mg}$ bid & 3 \\
\hline Amoxicillin-Clavulanate $^{a}$ & $500 / / 25 \mathrm{mg}$ tid & 7 \\
\hline Cefuroxime Axetil ${ }^{a}$ & $250-500 \mathrm{mg}$ bid & 7 \\
\hline Cefaclor $^{\mathrm{a}}$ & $250 \mathrm{mg}$ bid & 7-May \\
\hline Cefixime $^{a}$ & $400 \mathrm{mg}$ once & 7 \\
\hline Cefpodoxime-Proxetil ${ }^{2}$ & 100 mg bid & 7-Mar \\
\hline Ceftibuten $^{\mathrm{a}}$ & $400 \mathrm{mg}$ once & 7-May \\
\hline Trimethoprim-Sulfamethoxazole & $160 / 800 \mathrm{mg}$ bid & 3 \\
\hline Trimethoprim ${ }^{\mathrm{d}, \mathrm{e}}$ & $100-150 \mathrm{mg}$ bid & 3 \\
\hline
\end{tabular}

bid:Twice daily; qid: Four times daily; tid:Thrice daily.

a. These antibiotics present nob clear risk to the fetus, on the basis of studies in animals, humans, or both (pregnancy category B).

b. Dose and duration taken from reference 14 .

c. Administer with meals. Manufacture's in Spain recommends $100 \mathrm{mg}$ tid.

d. These antibiotics have shown an adverse effect on the fetus on the basis of studies in animals (pregnancy category $\mathrm{C}$ ); use only if the potential benefit justifies the potential risk to the fetus.

e. Trimethoprim is commercialized in Spain as $160 \mathrm{mg}$ tablets. 
Fosfomycin trometamol is administered solely for therapy of acute uncomplicated cystitis as a single $3 \mathrm{~g}$ dose and is not indicated for pyelonephritis. ${ }^{1-25}$ It is best absorbed if given before food intake. It achieves high concentration in the urine and maintains high levels for over 24 hours. ${ }^{23-25}$ Several clinical investigations have compared the clinical and microbiologic efficacy of fosfomycin with respect to other first line antimicrobials for uncomplicated cystitis. The clinical efficacy of one $(3 \mathrm{~g})$ dose of fosfomycin ( $91 \%$ cure) is comparable to nitrofurantoin (93\%), trimethoprim-sulfamethoxazole (93\%) and fluoroquinolones $(90 \%)$ in acute uncomplicated cystitis. ${ }^{1}$ The microbiologic cure rate of fosfomycin (80\%) is lower than comparable antibiotics at $88 \%$ to $94 \%{ }^{1-6}$ However, a recent meta-analysis of 27 trials found no difference in efficacy between fosfomycin and other antibiotics for the treatment of cystitis and found that fosfomycin was associated with significantly fewer adverse reactions in pregnant women. ${ }^{31}$ In addition, in a recent randomized single-blinded study, single dose fosfomycin at $3 \mathrm{~g}$ was as effective as ciprofloxacin, at 500 $\mathrm{mg}$ twice a day for 5 days, in the treatment of acute uncomplicated cystitis. $^{32}$

Additional usefulness of fosfomycin is in the treatment of multidrug-resistant organisms. Several in vitro studies have demonstrated that fosfomycin is active against vancomycin-resistant Staphylococcus aureus, and ESBL-producing Gram-negative rods. ${ }^{1-25}$ Two studies evaluated oral treatment with fosfomycin trometamol for lower urinary tract infections with ESBL-producing E. coli, either as a $3 \mathrm{~g}$ single-dose or once every other nigtht for three days. Treatment with fosfomycin was associated with clinical cure in 75 of the $80(93.8 \%)$ patients included in these two studies. ${ }^{29,30}$ In adition, observational studies have also shown that fosfomycin can be effective in the treatment of urinary tract infections due to $K$. pneumoniae carbapenamase-producing Enterobacteriaceae with 3-g doses repeated every 48 to 72 hours. ${ }^{25}$ Therefore, as resistance among uropathogens causing community-acquired uncomplicated cystitis increases, fosfomycin may become more useful, particularly if no other oral agents with in vitro activity are available. ${ }^{1-25}$

Finally, fosfomycin appears to have minimal propensity for collateral damage. Although the effect of fosfomycin on the intestinal flora after intake of a single 3-g dose has not been well studied, the effect is probabley minor. ${ }^{1}$ This asumption is suspected by the high rate of $E$. coli susceptibility in regions with frequent use of fosfomycin for uncomplicated cystitis in women. ${ }^{1}$ The convenience of a single-dose regimen, in vitro activity against resistant Gram-negative rods, and minimal propensity for collateral damage make fosfomycin a useful choice in the treatment of acute uncomplicated cystitis, particularly in areas, like Spain, where other antimicrobials are no longer useful for empirical therapy due to an unacceptable rate of resistances. The European Association of Urology and the Infectious Diseases Society of America list fosfomycin as first-line therpay for uncomplicated cystitis in women, despite it might be slightly less efective than other agents. ${ }^{1}$ Recently, the Spanish Society of Clinical Microbiology and Infectious Diseases (SEIMC) has also considered fosfomycin as first-line therpay for uncomplicated cystitis. ${ }^{8}$ Thus, fosfomycin trometamol ( $3 \mathrm{~g}$ in a single dose) is an appropriate choice for therapy in uncomplicated cystitis due to minimal resistance and propensity for collateral damage, but it appears to have a slightly inferior efficacy compared with other satadard short-course regimens (A-I).

\section{Nitrofurantoin}

Nitrofurantoin, belonging to a group of synthetic nitrofuran, was initially introduced as a microcrystalline form. In 1967 a macrocrsitalline form with improved gastrointetinal tolerance became available. ${ }^{20-33}$ Nowadays, there are two basic presentations of nitrofurantoin: the macrocrystalline form and a mixture of the microcrystalline and macrocrystalline forms $(25 \mathrm{mg}$ macrocristals plus $75 \mathrm{mg}$ monohydrate form), ${ }^{25-33}$ blended in a patented dual delivery system, which is known in USA as nitrofurantoin monohydrate/macrocristals. ${ }^{34}$ For therapy of UTIs nitrofurantoin in the macrocrystalline formulation is given orally at 50 to $100 \mathrm{mg}$ four times daily. ${ }^{20-25}$ The dose of the mixture of microcrystalline and macrocrystalline formulation is $100 \mathrm{mg}$ twice a day. ${ }^{25-34}$ This latter presentation is not commercialized in Spain.

The mechanisms of bacterial activity appears to involve multiple sites, including inhibition of ribosomal translation, bacterial DNA damage, and interference with the Krebs cycle. Nitrofurantoin is active against more tan $90 \%$ of $E$. coli strains causing UTI. ${ }^{4-25}$ Resistance to nitrofurantoin is uncommon, probably because of the multiple sites of action of the drug. However, Proteus, Serratia and Pseudomonas have natural resistance to nitrofurantoin. ${ }^{25}$ Enterococci, including those vancomycin-resistant, are susceptible to nitrofurantoin. $S$. aureus and $S$. saprophyticus are usually susceptible. ${ }^{25}$ Nitrofurantoin could also be a further option for oral antimicrobial treatment of acute uncomplicated cystitis produced by ESBL-producing bacteria. ${ }^{30}$ However, there is a growing concern of increasing resistance of ESBL-producing organisms against nitrofurantoin, particularly in hospital-acquired infections. ${ }^{15}$

Absorption is improved when nitrofurantoin is taken with food..$^{25}$ Serum concentrations of nitrofurantoin are low or undectectable with standard oral doses, as are concentrations in prostatic secretions to be used in patients with prostate infections. ${ }^{25-33}$ The antibiotic is eliminated predominatly in urine, where the drug concentration (50 to $250 \mu \mathrm{g} / \mathrm{mL}$ ) easily exceeds the MIC of $32 \mu \mathrm{g} / \mathrm{mL}$. Nitrofurantoin should not be administered to patients with substantial renal insufficiency (creatinine clearance $<60 \mathrm{~m} / \mathrm{min}$ ) ${ }^{25-34}$ but Cunha et al. ${ }^{35}$ has recently observed that the drug is highly effective in patients with creatinine clearance of $60-30 \mathrm{~mL} / \mathrm{min} .{ }^{35}$ Nitrofurantoin has been used safely in pregnant women and children. ${ }^{2-25}$ Past studies with 3 days of treatment with nitrofurantoin demonstrated persistence of the pathogen in the periurethral, vaginal, and rectal areas, so a 7-day course was recommended. ${ }^{1-33}$ In a randomized clinical trial Gupta et al. ${ }^{36}$ showed that a 5-day regimen of nitrofurantoin was comparable with 3 days of trimethoprim-sulfamethoxazole in the treatment of women with cystitis. ${ }^{36}$

Thus, most authorities are in agreement with the recommendation of a 5-day course of nitrofurantoin for the treatment of acute uncomplicated cystitis. In earlier non comparative studies bacteriologic and clinical responses in patietns with acute uncomplicated cystitis ranged from 61 to 100 percent. ${ }^{20}$ More recent studies have indicated an early clinical cure rate with nitrofurantoin of $79 \%$ to $95 \%$ and a microbiologic cure rate of 79 to $92 \% .^{1-33}$ Taken together, clinical efficacy studies indicate an overall equivalence between nitrofurantoin given for 5 or 7 days and trimethoprim-sulfamethoxazole, ciprofloxacin and single-dose fosfomycin trometamol. However, microbiological cure rate consistently showed a slightly more favorable effect for comparators. ${ }^{25-33}$ Nitrofurantoin is now considered a first-line therapeutic agent for acute uncomplicated cystitis because of the efficacy of a 5-day course and little risk for collateral damage to the normal human flora. ${ }^{1-8}$ Nitrofurantoin should not be used for treatment of pyelonephritis. ${ }^{1-33}$ Nitrofurantoin macrocrystals (50-100 
mg 4 times daily for 5 days) is an appropriate choice for therapy of acute uncomplicated cystitis, due to minimal resistance and propensity for collateral damage and efficacy comparable to other antimicrobials agents used for treatment (A-I).

\section{Fluoroquinolones}

Several studies have demonstrated the efficacy of fluoroquinolones in the tratment of UTI. Overall clinical and bacterial efficacy rates in the studies are consistently high, although they wer occasionally $<90 \%$. ${ }^{1}$ A recent meta-analysis study has shown that fluoroquinolones (ciprofloxacin and gatifloxacin) are the most effective therapy for uncomplicated UTI in comparison with other antimicrobials. ${ }^{37}$ According to a Cochrane analysis all fluoroquinolones (at comparable dosages) suitable for the treatment of UTI showed the same effectiveness for this indication but tolerability differed. ${ }^{38}$ Thus, fluoroquinolones remain very effective for the treatment of acute cystitis, although increased fluoroquinolone resistance among community uropathogens is limiting the usefulness of this antimicrobial class. ${ }^{1-37}$ Single-dose fluoroquinolone therapy may be an option for actue uncomplicated cystitis but with possibly lower efficacy rates than with 3-day regimens. On the other hand, an extended released formulation of ciprofloxacin $(500 \mathrm{mg}$ oral once daily) was comparable to conventional ciprofloxacin dose (250 mg PO twice daily) when both were given for three days. ${ }^{1}$ This formulation is not available in Spain.

The main concern with respect to fluoroquinolones use in acute cystitis is the appearance of resistance, not only among uropathgens but also other organisms, causing more serious and difficult-to-treat infections at other sites, including meticillin resistant $S$. aureus (MRSA). ${ }^{1}$ However, not all experts agree with this contention. ${ }^{2}$ They believe that a short course of fluoroquinolones for cystitis probably adds little pressure to spread of resistance compared with the widespread not indicated use of fluoroquinolones. In any case, in a recent systematic review and meta-analysis the authors concluded that fluoroquinolones have no added value over other antibitoic groups (nitrofurantoin, beta-lactams and trimthoprim-sulfamthoxazole) for the treatment of acute uncomplicated UTI. ${ }^{19}$ Moreover, the U.S. Food and Drug Administration advises that the serious side effects assoicated with fluoroquinolone antibarerial drugs generally otuweigh the benefits for patients with non serious infections, such as uncomplicated urinary tract infections. ${ }^{39}$ The fluoroquinolones (ciprofloxacin, levofloxacin, norfloxacin and ofloxacin) are highly efficacious in 3-day regimens (A-I) but should be considered alternative antimicrobials for acute uncomplicated cystitis, because they have a high propensity for collateral damage (B-III).

\section{$\beta$-Lactams}

Pivmecillinam (or amdinocillin pivoxil) is an orally active prodrug of mecillinam, an extended spectrum penicillin, which is used exclusively for treatment of lower UTI. In the IDSA guidelines, it was proposed as a first-line drug for empirical treatment of uncomplicatd cystitis, because of minimal resistance and propensity for collateral damge. However, neither pivmecillinam nor mecillinam are licensed in Spain. Ampicillin and amoxicillin can no longer be recommended as empirical treatment in acute uncomplicated cystitis given the relatively poor efficacy and the very high prevalence of antimicrobial resistance to these agents worldwide. The most used $\beta$-lactams for the treatment of uncomplicated UTI have been amoxicillin-clavulanate and oral cephalosporins. The oral cephalosporins of second generation (cefuroxime axetil, cefaclor) have imporved activity against E.coli compared with those of first generation (cepahlexin, cefadroxil), but lower activity compared with those of third generation (cefdinir, cefditoren pivoxil, cefixime, cefpodoxime proxetil and ceftibuten).

In the ARESC study the overall susceptibility pattern of E.coli and the total of uropathogens for cefuroxime was very similar to that of amoxicillin-clavulanate. Cefdinir, a widely used cephalosporin in United States, is not commercialized in Spain. Most studies demonstrate that $\beta$-lactams are generally inferior in cure rate to the fluoroquinolones and TMP-SMX. A recently published randomized controlled trial on cefpodoxime, a before promising oral cephalosporin, by Hooton and co-workers has showed that cefpodoxime was inferior to ciprofloxacin..$^{40}$ In addition, a recent meta-analysis study showed the lowest efficacy of amoxicillin-clavulanate in comparison with other antibitoics for the treatment of acute cistitis. ${ }^{37}$ One possible explanation for this inferiority is that use of $\beta$-lactams is associated with a lower rate of eradication of vaginal uropathognes, persisting the reservoir for infection. Additionally, amoxicillin clavulanic acid may be useful in the therapy of patients with cystitis due to ESBLproducing Enterobacteriacea. Rodriguez-Baño et al..$^{29}$ used amoxicillin clavulanate $(500 \mathrm{mg} / 125 \mathrm{mg}$ thrice daily, for 5-7 days) in a series of 37 patients with cystitis caused by ESBL-producing E. coli and it was associated with a favourable overall cure rate of $84 \%$.

Broad-spectrum cephalosporins, in particular, have been associated with collateral damage, the most concerning of which is ESBL resistance among Gram-negative bacteria. Therefore, experts advise avoidance of $\beta$-lactams for empirical therapy of uncomplicated cystitis unless none of the first-line recommended agents are appropriate. If the use of one $\beta$-lactam is chosen, the duration of treatment should be of seven days, since a shorter course is not adequate for -lactams antibiotics. $\beta$-lactam agents other than pivmecillinam, including amoxicillin-clavulanate, cefaclor, ceftibuten and cefpodoximeproxetil in 7-day regimen are appropriate choices for therapy when other recommended agents cannot be used (B-I). The $\beta$-lactams generally have inferior efficacy and more adverse effects, compared with other UTI antimicrobial (B-I). Ampicillin and amoxicillin should not be used for empirical teatment of uncomplicated cystits, given the high prevalence of antimicrobial resistance to these agents (E-II).

\section{Trimethoprim-sulfamethoxazole (TMP-SMX)}

TMP-SMX (160/800 mg [1 double-strength] twice-daily for 3 days) is a highly effective antimicrobial for therapy of acute uncomplicated cystitis, with clinical and microbiological cure rates ranging between $86 \%$ to $100 \%$ and $85 \%$ to $100 \%$, respectively. Moreover, it is not thought to have an important propensity for collateral damage, as it is observed with broad-spectrum cephalosporins or fluoroquinoles. Thus, it was recommended as a first-line agent in the IDSA guideliens for uncomplicated cystitis when the rate of resistance is expected to be $<20 \%$. Even, in settings with a $10-15 \%$ prevalence of resistance to trimethoprim-sulfamethoxazole, cure rates with this antimicrobial were equivalent to those with comparative drugs (i.e., ciprofloxacin and nitrofurantoin). Previous data had shown that trimethoprim (100 $\mathrm{mg}$ twice daily for 3 days) was equivalent to its combination with sulfamthoxazole for the treatment of acute uncomplicated cystitis.

In Spain, the reported resistance pattern of E. coli to TMP-SMX has varied between $27 \%$ to $34 \%,{ }^{4-16}$ making impractical this agent to be used as empirical treatment in acute uncomplicated cystitis. Nevertheless, TMP-SMX is an excellent antimicrobial for acute uncomplicated UTI in cases known to be caused by a susceptible strain. In Sapin, trimethoprim-sulfamthoxazole is not recommended 
for empirial treatment of acute cystitis, because the resistance prevalence of $E$. coli is higher than $20 \%$ in our country (E-I). On the other hand, if the infecting organism is known to be susceptible to trimethoprim-sulfamethoxazole this agent is a very effective therapy (A-I).

\section{Conclusion}

In an excellent systematic review, the randomized controlled trials of different classes of antibiotics for the treatment of acute uncomplicated UTI were analysed. Antimicrobials included nitrofurantoin, fluoroquinolones, $\beta$-lactams and TMP-SMX, all of them administered, at least, for three days. Primary outcomes were early and late clinical cure; and secondary otucomes were bacteriological cure and the appearance of adverse side effects with the drugs. Early and late clinical cures were defined as the absence of urinary symptoms at 7-10 days and 30 days after the last dose of antimicrobial, respectively. The authors found no differences for the symptomatic cure between the different classes of antimicrobials included in their study. Fewer rashes were observed in patients treated with fluoroquinolones than with either $\beta$-lactam drugs or TMP-SMX, but the risk for any advere event was similar. Likewise, nitrofurantoin was less likely to cause rash tan TMP-SMX, while having similar rates for any adverse event.

In the event of diagnostic uncertainty regarding cystitis versus early pyelonephritis, use of agnets such as nitrofurantoin and oral fosfomycin should be avoided, because they do not achieve adequate renal tissue levels. Such uncertainty may exist in the setting of cystitis symptoms accompanied by subjective fever that is not verified at the time of examination, a prolonged duration of cystitis symptoms (typically greater than 5-7 days), or vague flank pain or tenderness which is not otherwise explained. Finally, as it was stated in the IDSA guidelines, "the choice of agent should be individualized on the basis of patient allergy and compliance history, local practice patterns, local community resistance prevalence, availability, cost, and patient and provider threshold for failure".

If a first-line antimicrobial agent (fosfomycin or nitrofurantoin) is not a good choice on the basis of one or more of these factors, fluoroquinolones or beta-lamctams are reasonable alterantives although it is preferable to minimize their urse because of concerns about ecologic adverse effects (and efficacy in the case of betalactams). Beta-lactams are less effective than fluoroquinolones and TMP-SMX for treatment of acute cystitis. However, because of concerns about the aderse effects from fluoroquinolones, the riskbenefit balance for acute cystitis favors the use of fluoroquinolones only if other agents (including -lactams) cannot be used.

\section{Acknowledgements}

None.

\section{Conflict of interest}

The author declares no conflict of interest.

\section{References}

1. Gupta K, Hooton TM, Naber KG, et al. International clinical practice guidelines for the treatment of acute uncomplicated cystitis and pyelonephritis in women: a 2010 update by the Infectious Diseases Society of America and the European Society of Microbiology and Infectious Diseases. Clin Infect Dis. 2011;52(5):103e-120e.
2. Sobel JD, Kaye D. Urinary tract infections. In: Bennett JE, Dolin R, et al. editors. Mandell, Douglas and Bennett's principles and practice of infectious diseases. 8th ed. Philadephia, Saunders, USA; 2015. p. 886913.

3. Hooton TM, Gupta K. Acute uncomplicated cystitis and pyelonephritis in women. 2017.

4. Palou J, Pigrau C, Molina I, et al. Etiología y sensibilidad de los uropatógenos identificados en infecciones urinarias bajas no complicadas de la mujer (Estudio ARESC): implicaciones en la terapia empírica. Med Clin. 2011;136(1):1-7.

5. Takhar SS, Moran GJ. Diagnosis and management of urinary tract infection in the Emergency Department and outpatient settings. Infect Dis Clin N Am. 2014;28(1):33-48.

6. Grigoryan L, Trautner BW, Gupta K. Diagnosis and management of urinary tract infections in the outpatient setting. A review. JAMA. 2014;312:1677-1684.

7. Naber KG, Wullt B, Wagenlehner FME. Antibiotic treatment of uncomplicated urinary infection in premenopausal women. Int $J$ Antimicorb Agents. 2011;38(Supp):21-35.

8. de Cueto M, Aliaga L, Alós JI, et al. Executive summary of the diagnosis and treatment of urinary tract infection: Guidelines of the Spanish Society of Clinical Microbiology and Infectious Diseases (SEIMC). Enf Infecc Microbiol Clin. 2017;35(5):314-320.

9. Schito GC, Naber KG, Botto H, et al. The ARESC study: an international survey on the antimicrobial resistance of pathogens involved in uncomplicated urinary tract infections. Intern $J$ Antimicrob Agents. 2009;4:407-413.

10. Naber KG, Schito G, Botto H, et al. Surveillance study in Europe and Brazil on clinical aspects and antimicrobial resistance epidemiology in females with cystitis (ARESC): implications for empiric therapy. Eur Urol. 2008;54:1164-1178.

11. Heytens S, Boelens J, Claeys G, et al. Uropathogen distribution and antimicrobial susceptibility in uncomplicated cystitis in Belgium, a high antibiotics prescribing country: 20-year surveillance. Eur J Clin Microbiol Infect Dis. 2017;36(1):105-113.

12. Hooton TM, Roberts PL, Cos ME, et al. Void midstream urine culture and acute cystitis in premenopausal women. $N$ Engl $\mathrm{J} \mathrm{Med}$. 2013;369(20):1883-1891.

13. Gupta K, Hooton TM, Miller L. Managing uncomplicated urinary tract infection-making sense out of resistance data. Clin Infect Dis. 2011;53(10):1041-1042.

14. Etienne M, Lefebvre E, Frebourg N, et al. Antibiotic treatment of acute uncomplicated cystitis base on rapid urine test and local epidemiology: lessons from a primary care series. BMC Infect Dis. 2014;14:137.

15. Bader MS, Loeb M, Brooks AA. An update on the management of urinary tract infections in the era of antimicrobial resistance. Post Med. 2017; 129(2):242-258.

16. Cuevas O, Cercenado E, Gimeno M, et al. Comparative in vitro activity of cefditoren and other antimicrobials against Enterobacteriaceae causing community-acquired uncomplicated urinary tract infections in women: a Spanish nationwide multicenter study. Diagn Microbiol Infect Dis. 2010;67(3):251-260.

17. Katsarolis I, Poulakou G, Athanasia S, et al. Acute uncomplicated cystitis: from surveillance data to a rationale for empirical treatment. Int $J$ Antimicrob Agents. 2010;35(1):62-67.

18. Gupta K, Hooton TM, Stamm WE. Increasing antimicrobial resistance and the management of uncomplicated community-acquired urinary tract infections. Ann Intern Med. 2001;135(1):41-50. 
19. Zalmanovici Testioreanu A, Green H, Paul M, et al. Antimicrobial agents for treating uncomplicated urinary tract infection in women. Cochrane Database Syst Rev. 2010;10:CD007182.

20. Hooper DC. Urinary tract agents: nitrofurantoin and methenamine. In Mandell GL, Bennett JE, et al. editors. Mandell, Douglas and Bennett's principles and practice of infectious diseases. 4th ed. New York, USA 1995. p. 376-381.

21. Katchman EA, Milo G, Paul M, et al. Three-day vs longer duration of antibiotic treatment for cystitis in women: systematic review and metaanalysis. Am J Med. 2005;118(11):1196-1207.

22. Milo G, Katchman EA, Paul M, et al. Duration of antibacterial treament for uncomplicated urinary tract infection in women (Review). Cochrane Database of Systematic Reviews. 2005;2:CD004682.

23. Popovic M, Stenot D, Pillai S, et al. Fosfomycin: an old, new friend? Eur J Clin Microbiol Infect Dis. 2010;29(2):127-142.

24. Michalopoulos AS, Livadites IG, Gougoutas V. The revival of fosfomycin. Int J Infect Dis. 2011;15(11):e732-739.

25. Horton JM. Urinary tract agents: nitrofurantoin, fosfomycin, and methenamine In: Bennett JE, Dolin R, editors. Douglas, Mandell and Bennett's principles and practice of infectious diseases. 8th edition. Philadelphia: Elsevier, USA; 2015. p. 447-451.

26. Saiprasad PV, Krishnaprasad K. Exploring the hidden potential of fosfomycin for the fight against severe Gram-negative infections. Indian J Med Microbiol. 2016;34(4):416-420.

27. Matsumoto T, Muratami T, Nakahama C, et al. Clinical effects of 2 days treatment by fosfomycin calcium for acute uncomplicated cystitis in women. J infect Chemother. 2011;17(1):80-86.

28. Patwardhan V, Singh S. Fosfomycin for the treatment of drug-resistant urinary tract infections: potential of an old drug not explored fully. Int Urol Nephrol. 2017;2(1):100.

29. Rodríguez Baño J, Alcalá JC, Cisneros JM, et al. Community infections caused by extended-spectrum $\beta$-lactamase-producing Escherichia coli. Arch Intern Med. 2008;168(17):1897-1902.

30. Falagas M, Kastoria AC, Kapaskelis AM, et al. Fosfomycin for the treatment of multidrug-resistant, including extended-spectrum $\beta$ lactamase producing Enterobacteriaceae infections: a systematic review. Lancet Infect Dis. 2010;10(1):43-50.
31. Falagas ME, Vouloumanou EK, Togias AG, et al. Fosfomycin versus other antibiotics for the treatment of cystitis: a meta-analysis of randomized controlled trials. J Antimicrob Chemther. 2010;65(9):1862-1877.

32. Ceran N, Mert D, Kocdogan FY, et al. A randomized comparative study of single-dose fosfomycin and 5-day cirpofloxacin in female patients with uncomplicated lower urinary tract infections. J Infect Chemother. 2010;16(6):424-430.

33. Huttner A, Verhaegh EM, Harbarth S, et al. Nitrofurantoin revisited: a systematic review and meta-analysis of controlled trials. J Antimicrob Chemother. 2015;70(9):2456-2464

34. Spencer RC, Moseley DJ, Greensmith MJ. Nitrofurantoin modified release versus trimethoprim or co-trimoxazole in the treatment of uncomplicated urinary tract infection in general practice. $J$ Antimicrob Chemother. 1994;33(suppl A):121.129.

35. Cunha BA, Cunha CB, Lam B, et al. Nitrofurantoin safety and effectiveness in treating acute uncomplicated cystitits (AUC) in hospitalized adults with renal insufficiency: antibiotic sterwardship implications. Eur J Clin Microbiol Infect Dis. 2017;36(7):1213-1216.

36. Gupta K, Hooton TM, Roberts PL, et al. Short-course nitrofurantoin for the treatment of acute uncomplicated cystitis in women. Arch Intern Med. 2007;167(20):2207-2212.

37. Knottnerus BJ, Grigoryan L, Geerlings SE, et al. Comparative effectiveness of antibiotics for uncomplicated urinary tract infections: network meta-analysis of randomized trials. Fam Pract. 2012;29(6):659 670 .

38. Rafalsky V, Andreeva J, Rjabkova E. Quinolones for uncomplicated acute cystitis in women. Cochrane Database Sust Rev. 2006;19(3):CD003597.

39. US Food and Drug Administration. FDA Drug Safety Communication: FDA advises restricting fluoroquinolone antibiotic use for certain uncomplicated infections; warns about disabling side effects that can occur together, USA;

40. Hooton TM, Roberts PL, Stapleton AE. Cefpodoxime vs ciprofloxacin for short-course treatment of acute uncomplicated cystitis. JAMA. 2012;307(6):583-589. 